\title{
Estado actual de los proyectos colaborativos educativos en Twitter
}

\author{
Camino LóPEz GARCíA \\ Universidad de Salamanca \\ camino_lopez@usal.es \\ M Cruz SÁNCHEZ GóMEZ \\ Universidad de Salamanca. \\ mcsago@usal.es \\ Beatriz Palacios Vicario \\ Universidad Pontificia de Salamanca \\ bpalaciosvi@upsa.es
}

\section{Resumen}

Esta investigación pretende comprobar la influencia que los proyectos colaborativos en Twitter están teniendo en la comunidad educativa online. Especialmente, buscamos el proyecto colaborativo que más influencia tiene en esta red social. Para ello, hemos utilizado un programa de medición de la influencia online, llamado Topsy, estudiando los datos del hastag y cuenta del proyecto colaborativo, bajo una metodología ex post facto en un estudio de desarrollo que pretende observar las variables durante un periodo de tiempo determinado.

Palabras clave: proyectos colaborativos, Twitter, redes sociales, Topsy, profesores, influencia.

\section{Current status of educational collaborative projects on Twitter.}

\begin{abstract}
This research aims to test the influence that on Twitter collaborative projects are having on the online education community. Especially, we seek collaborative project greatest influence on this network. To do this, we used a program online influence measurement, called Topsy, studying hastag data and collaborative project account under an ex post facto methodology development study aimed to observe the variables over a period of time.
\end{abstract}

Key words: collaborative projects, Twitter, social networks, Topsy, teachers, influence

\section{Referencia normalizada:}

López García, C.; Sánchez Gómez, M. C. y Palacios Vicario, B. (2013) Estado actual de los proyectos colaborativos educativos en Twitter. Historia y Comunicación Social. Vol. 18. N ${ }^{\circ}$ Especial Diciembre. Págs. 733-751.

Sumario: 1. Introducción 2.Estado de la cuestión y objetivo de la investigación 3.Metodología 4. Los proyectos colaborativos en red 5. El Barco del Exilio 6. Conclusiones 7. Bibliografía 


\section{Introducción}

Las Nuevas tecnologías no solo han supuesto un cambio en la sociedad sino que su impacto está teniendo una gran repercusión en el sector educativo. Las redes sociales en concreto, han abierto nuevos canales de conversación (Siemens, G; 2004) y colaboración entre profesores especialmente interesados en integrar las TIC en su práctica educativa. Es esta la razón de una nueva tendencia que no hace más que ir en aumento: la creación de proyectos colaborativos en red (Suárez, C. y Gros, B.; 2013).

Twitter es una red social que especialmente atiende a las necesidades de los profesores: conectividad, inmediatez, brevedad y amplitud. Los docentes más preocupados por la integración de las TIC en su práctica docente, tienen presencia en esta red social, en la cual encuentran colaboración por parte de otros iguales, compartiendo sus experiencias, nuevas ideas y trabajo. No todos buscan reconocimiento, la gran mayoría utilizan esta forma de comunicarse para subirse a las alas de la innovación educativa, preocupados por la calidad de la enseñanza de sus alumnos. En estos espacios pueden autoformarse, manteniéndose actualizados, aprendiendo entre pares. El poder de las redes sociales es la influencia que genera la actividad de los perfiles que en ella anidan, tanto en positivo como en negativo (Fowler, 2011)

En el caso de la comunidad educativa, la influencia de los perfiles de profesores innovadores en Twitter genera un interés creciente en los demás docentes que no poseen tantas destrezas tecnológicas o que no se mantienen tan actualizados, ya que se sienten inspirados y amparados por toda la información y conocimiento que los primeros comparten altruistamente en la red social (Meirinhos, M. y Asorio, A.; 2009). Se trata de un ir y venir de pequeñas píldoras en forma de 140 caracteres, que son los permitidos por mensaje en Twitter, también llamado tuit. La ventaja de aportar información en un espacio tan resumido es la capacidad para centrar el interés en el punto más relevante, utilizando el resto de caracteres para enriquecer el mensaje con un vínculo a un espacio de la red en el que se encuentre más información acerca del tema en cuestión y un hastag o nube de conversación sobre el tema.

Es en este contexto en el que muchos proyectos colaborativos educativos propuestos por docentes, ven la luz y se desarrollan, alimentándose de las colaboraciones crecientes de la comunidad educativa online.

\section{Estado de la cuestión y objetivo de la investigación}

En estos entornos virtuales, los profesores son los que toman la iniciativa sobre su propio trabajo y el avance del mismo, integrando en sus perspectivas de aprendizaje y colaboración a otros profesores igual de motivados. Precisamente, por esta razón surgió el documento que recoge los proyectos colaborativos online. El profesor Gregorio Toribio, un gran conocedor de la snuevas tecnologías y la educación, tomó esta iniciativa que fue acogida en el marco del servicio Curalia, espacio dedicado a 
recopilar buenas prácticas educativas reales que han tenido o tienen un impacto en la comunidad educativa. Estas experiencias pueden ser tanto del entorno formal como invisible, es decir, sin programación de un aprendizaje reglado (Cobo, C. y Moravec, J.; 2011). Este espacio pertenece a la Fundación Telefónica para la Educación, la cual lo creó en 2012.

El documento que recoge los proyectos colaborativos online lleva por nombre Propuestas de proyectos colaborativos. Está dividido en diversas secciones para procurar recoger la mayor cantidad de datos sobre el proyecto que se integra en este espacio recopilador. Se requiere información acerca de las personas participantes en el mismo, sus perfiles de Twitter, un email de contacto, el título, el nivel educativo al que están dirigidos, un espacio web que recoja la iniciativa y el hastag que ha decidido crear para crear conversaciones en Twitter.

En la actualidad son muchos los proyectos colaborativos online propuestos, no tantos los activos en este momento. Muchos de ellos, aunque están registrados en el documento colaborativo de referencia, ya se ha terminado o simplemente son abiertos en periodos específicos del año. Toda esta versatilidad está cambiando las metodologías de interacción entre los profesores y su forma de aprender. Según Suárez y Gros (2013), aprender en red tiene un impacto en el rol que afrontan los profesores. En muchos casos, este rol es de transmisor de contenidos y evaluador, pero para aprender en red entre iguales, deben asumir además que el otro es un agente educativo válido, por lo que también asumirá esos roles. A la vez el docente es discente, y el discente docente.

El proceso de integración de las TIC en la práctica educativa no solo es un proceso técnico, sino que requiere un cambio de aptitudes del profesorado (Cabero, J. 1994; Zhao y otros, 2002). Hay corrientes de pensamiento que consideran que los aprendizajes sustraídos de experiencias TIC son sesgados y superficiales, pero en cambio otros aseguran que se trata de aprendizajes significativos medidos por la personalización. Los Entornos Personales de Aprendizaje o PLE (Adell, J. y Castañeda,; 2011) tienen la capacidad de ser espacios no caducos, que se reinventan con el discente y que le acompañan a lo largo de la vida. El trabajo colaborativo en red aporta innovación, valores morales, información, evita duplicidades, mejora las probabilidades de viabilidad de proyectos y genera unidad social. Todas ellas son afirmaciones del portal oficial del proyecto Escuela 2.0, evidenciando los beneficios de los que disfrutan los profesores que trabajan colaborativamente en red. Se hace posible gracias a la creación de ecosistemas de aprendizaje (Freire, J. 2011) en los cuales una gran heterogeneidad de perfiles se interrelacionan.

Estas redes de aprendizaje social son integradas por personas que comparten aprendizajes en la red. Aquellas que el individuo en la red considera que son merecedoras de consolidar su aprendizaje a lo largo de la vida, pasan a formar parte de su red personal de aprendizaje, también llamada PLN (Churches, R,: 2009). 
En nuestra investigación, pretendemos determinar qué proyectos colaborativos educativos activos en Twitter son los más influyentes en la red, identificando a aquellos contactos claves en la difusión de la información en los hastag del proyecto.

\section{Metodología}

El documento recopilador de propuestas de proyectos colaborativos en red, alberga a fecha de la investigación, 25/09/2013, un registro de 82 proyectos colaborativos. Nuestro objetivo es determinar qué proyectos colaborativos en red activos son los más influentes, con lo cual, hemos realizado un seguimiento de estos 82 proyectos inscritos hasta determinar que tan solo 10 de ellos estaban activos en el momento de la investigación. Esta selección configura la muestra con la que hemos trabajado a partir de un estudio de desarrollo basado en metodología ex post facto. Esto ha sido así ya que pretendíamos realizar un análisis de la progresión de estos proyectos colaborativos desde sus inicios hasta la fecha de la investigación, observando las variables durante un periodo de tiempo determinado.

Los proyectos colaborativos que ha sido determinados como viables para la investigación a partir de la población especificada, son los siguientes:

Cuadro no 1: Selección de los proyectos colaborativos participantes en la investigación.

\begin{tabular}{|l|l|l|}
\hline \multicolumn{1}{|c|}{ Proyecto colaborativo } & \multicolumn{1}{c|}{ Cuenta en Twitter } & \multicolumn{1}{c|}{ Hastag en Twitter } \\
\hline Proyecte Espurn@ & $@$ espurna & \#espurna \\
\hline Cero en Conducta & @CeroenConducta0 & \#educaycine \\
\hline Callejeros literarios & $@$ callejarios & \#callejarios \\
\hline Lóva & $@$ proyectolova & \#inLÓVA2 \\
\hline Kuentalibros & @ kuentalibros & \#kuentalibros \\
\hline As nosas letras & $@$ asnosasletras & \#asnosasletras \\
\hline El Barco del Exilio & @exilioyregreso & \#exilioyregreso \\
\hline Ortografía y Cómic & $@$ ortografiacomic & \\
\hline FP en tu Móvil & $@$ fpentumóvil & \#fpentumóvil \\
\hline Queremos MusicArte & @MusicArte & \#QMusicArte \\
\hline
\end{tabular}

Fuente: elaboración propia.

La Universidad Católica San Antonio de Murcia (UCAM) efectuó un análisis de la actividad en Twitter para el que utilizó una de las herramientas más novedosas en investigación cuantitativa en red llamada Topsy. Esta herramienta tiene la característica de ser de Cloud Computing, o computación en la nube, que permite acceder mediante un usuario y contraseña a una cuenta propia en el propio software, almacenado en internet. Esto permite al investigador tener independencia entre el software 
y el hardware desde el que accede al mismo, minimizando la incompatibilidad entre ambos y reduciendo así las limitaciones tecnológicas que surgen en el trabajo con otras herramientas de medición.

Topsy permite evaluar los hastag y las cuentas en Twitter de los proyectos colaborativos participantes de la muestra. De este modo, podemos conocer el impacto que estos proyectos tienen en la comunidad virtual en diferentes momentos de su trayectoria, el dominio geográfico de cada uno y su impacto y exposición en la red social. Concretamente, estamos hemos estado interesadas en el conocimiento del índice de exposición máxima de estos proyectos, que es la suma de la actividad que tiene la cuenta de Twitter o el hastag del proyecto colaborativo más la influencia que han tenido sus seguidores y otros usuarios activos de la red durante un periodo de tiempo determinado. El rango de tiempo que se refleja en este estudio corresponde a una exposición a lo largo de 30 días.

Según Burgns y Burgess (2011) los hastag son entornos que facilitan la discusión entre grupos que necesitan estar conectados a través de sus seguidores. A partir de la comunidad que los proyectos generan, podemos obtener datos relevantes acerca del impacto de los mismos en la red social. Por ello, hemos decidido que además de estudiar la cuenta propia de cada proyecto colaborativo, también hemos incluido la medición de los hastag como espacios de conversación y colaboración a partir del proyecto.

Mostramos a continuación los datos seleccionados de los proyectos para proceder a su medición:

Cuadro $\mathbf{n}^{0}$ 2. Instrumento de codificación para la investigación de los proyectos colaborativos.

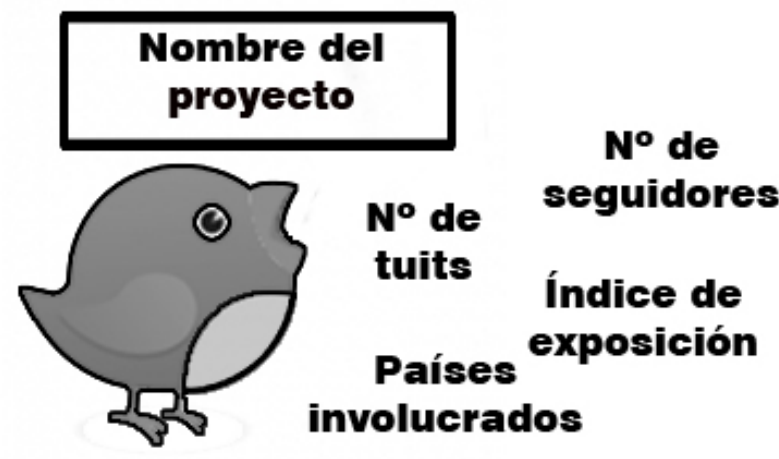

Fuente: elaboración propia.

Además, hemos tenido en cuenta determinadas variables categóricas como: los seguidores más influyentes del proyecto colaborativo, los seguidores influyentes con actividad de retuit y los de actividad de tuit. 


\section{Los proyectos colaborativos en red}

Los proyectos colaborativos en red son una rica fuente de datos que nos puede facilitar el conocimiento de cómo los educadores están haciendo uso de las redes sociales, y la información que en ellas comparten, dándonos una perspectiva fiable de la realidad educativa.

Estos proyectos colaborativos son creados por los propios docentes, a la medida de sus necesidades de desarrollo y temáticas de interés. A lo largo de estos 10 proyectos colaborativos que hemos estudiado, nos hemos encontrado con una gran variedad de temáticas y orientaciones. Todos ellos tienen en común que se iniciaron a partir del año 2010 y la media del índice de exposición es de 321,8.

El proyecto Espurn@ dio comienzo en mayo de 2013, y gracias a la investigación tenemos conocimiento de que ha seguido activo hasta septiembre de 2013. Su índice de exposición es inferior a la media de los proyectos colaborativos estudiados, que corresponde a 161, conseguido entre marzo y abril de 2011. La actividad de su hastag \#espurna fue iniciada previamente al comienzo del proyecto, en el 2009, lo cual nos indica que este nicho de conversación está influido por otro tipo de usuarios ajenos al proyecto. En este caso, no constituye una fuente fiable de información. En la siguiente tabla se puede apreciar una comparación entre la actividad del hastag y la del proyecto, viendo claramente que la línea de actividad de la cuenta del proyecto es plana cuando la del hastag ya realiza picos de movimiento.

Cuadro no 3. Tabla comparativa entre el hastag y la cuenta de Twitter del proyecto Espurn@.

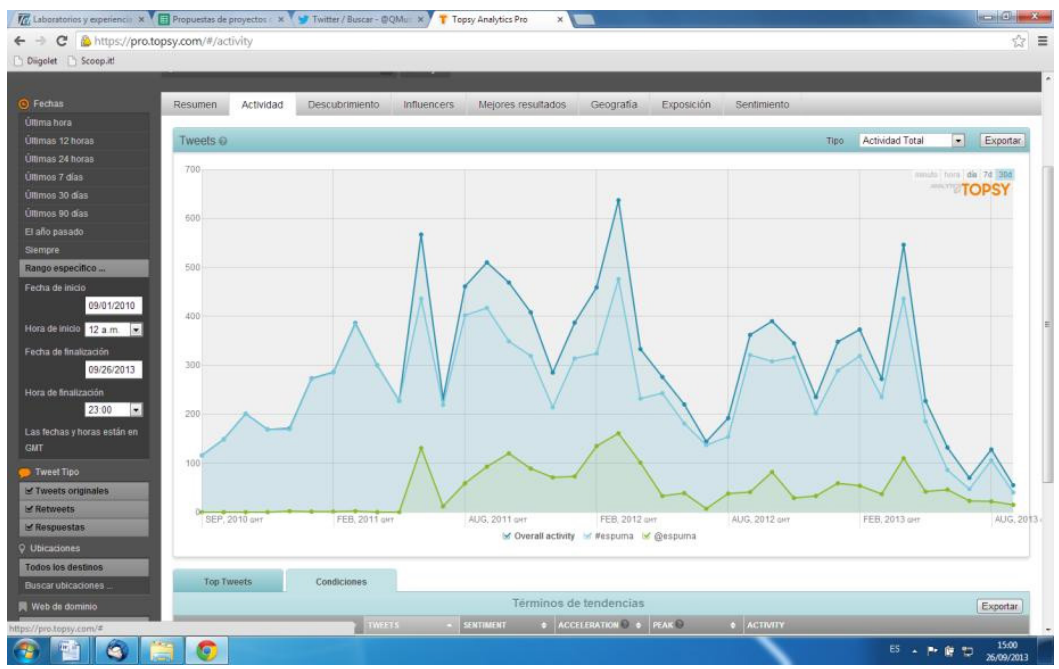

Fuente: elaboración propia. 
El proyecto colaborativo Cero en Conducta dio comienzo en junio de 2011, y gracias a la investigación tenemos conocimiento de que ha seguido activo actualmente. Su índice de exposición está dentro de la media de los proyectos colaborativos estudiados, que corresponde a 366, conseguido entre junio y julio de 2013. La actividad de su hastag \#educaycine fue iniciada en julio de 2012, un año después de dar comienzo la cuenta en Twitter del proyecto. En la siguiente tabla se puede apreciar una comparación entre la actividad del hastag y la del proyecto:

Cuadro $\mathbf{n}^{0}$ 4. Tabla comparativa entre el hastag y la cuenta de Twitter del proyecto Cero en Conducta.

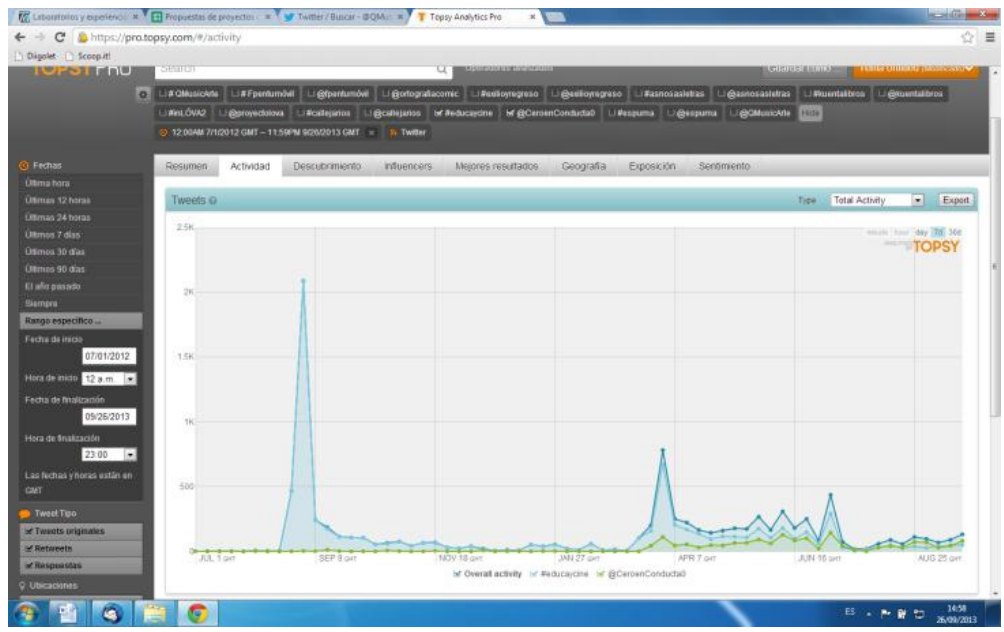

Fuente: elaboración propia.

El proyecto colaborativo Callejeros Literarios dio comienzo en diciembre de 2010, y gracias a la investigación tenemos conocimiento de que ha dejado de estar activo en junio de 2013. Este proyecto no se puede considerar inactivo ya que su actividad es especialmente a lo largo del curso lectivo. Su índice de exposición está por debajo de la media de los proyectos colaborativos estudiados, que corresponde a 159, conseguido entre mayo y junio de 2011. La actividad de su hastag \#callejarios fue iniciada en diciembre de 2010, al mismo tiempo que la cuenta en Twitter del proyecto. En la siguiente tabla se puede apreciar una comparación entre la actividad del hastag y la del proyecto: 
Cuadro ${ }^{0}$ 5. Tabla comparativa entre el hastag y la cuenta de Twitter del proyecto Callejeros Literarios.

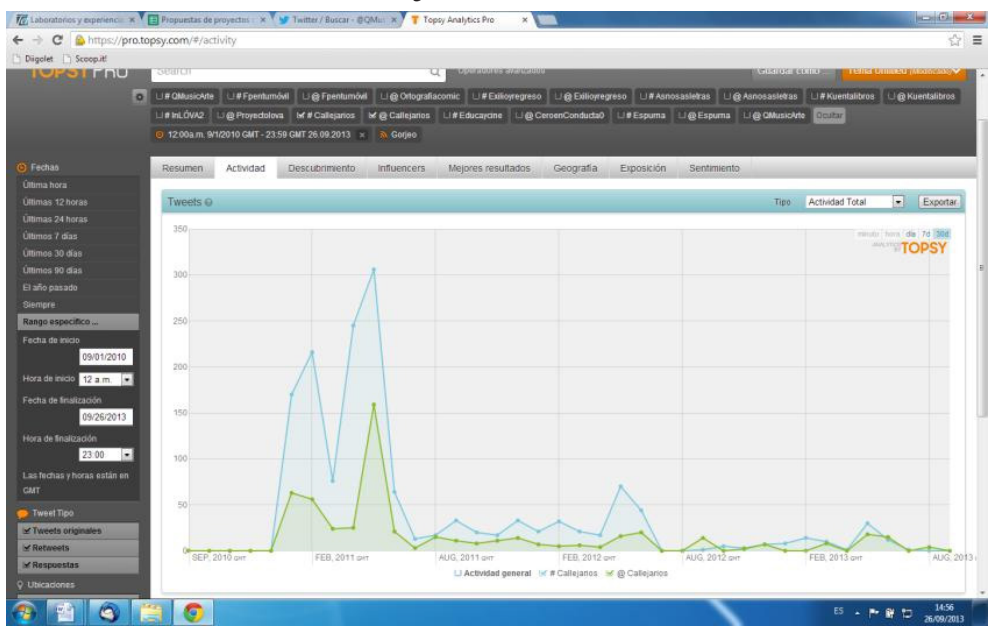

Fuente. Elaboración propia.

El proyecto colaborativo Lóva dio comienzo en diciembre de 2010, y gracias a la investigación tenemos conocimiento de que todavía está en funcionamiento. Su índice de exposición está por encima de la media de los proyectos colaborativos estudiados, que corresponde a 531, conseguido entre marzo y abril de 2012. La actividad de su hastag \#inLÓVA2 fue iniciada en enero de 2013, tres años más tarde del comienzo del proyecto en sí. En la siguiente tabla se puede apreciar una comparación entre la actividad del hastag y la del proyecto:

Cuadro $\mathbf{n}^{\mathbf{0}}$ 6. Tabla comparativa entre el hastag y la cuenta de Twitter del proyecto Lóva.

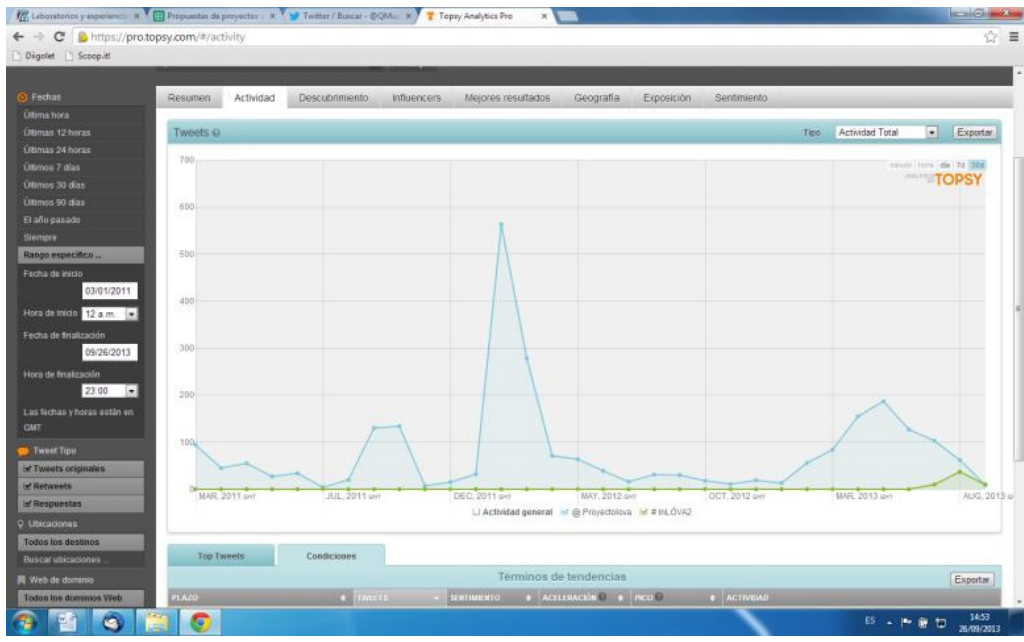

Fuente: elaboración propia. 
El proyecto colaborativo Kuenta Libros dio comienzo en mayo de 2011, y gracias a la investigación tenemos conocimiento de que todavía está en funcionamiento. Su índice de exposición está por encima de la media de los proyectos colaborativos estudiados, que corresponde a 503, conseguido entre octubre y noviembre de 2012 . La actividad de su hastag \#kuentalibros fue iniciada en abril de 2011, al mes siguiente de comenzar el proyecto. En la siguiente tabla se puede apreciar una comparación entre la actividad del hastag y la del proyecto:

Cuadro $\mathbf{n}^{0}$ 7. Tabla comparativa entre el hastag y la cuenta de Twitter del proyecto Kuenta Libros.

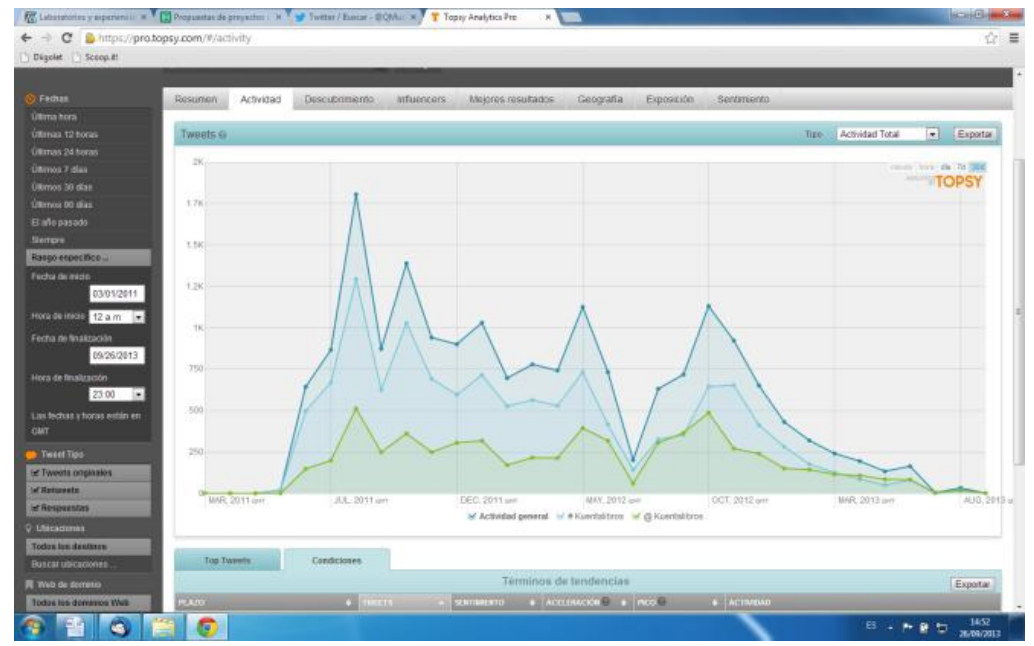

Fuente: elaboración propia.

El proyecto colaborativo As nosas letras dio comienzo en abril de 2011, y gracias a la investigación tenemos conocimiento de que todavía está en funcionamiento. Su índice de exposición está muy por debajo de la media de los proyectos colaborativos estudiados, que corresponde a 59, conseguido entre abril y mayo de 2013. La actividad de su hastag \#asnosasletras fue iniciada en marzo de 2011, un mes antes de dar comienzo la actividad de la cuenta de Twitter, lo que nos indica que el proyecto surgió de conversaciones directas en el hastag y que se hizo la necesidad de crear una cuenta propia del proyecto. En la siguiente tabla se puede apreciar una comparación entre la actividad del hastag y la del proyecto: 
Cuadro ${ }^{0}$ 8. Tabla comparativa entre el hastag y la cuenta de Twitter del proyecto As nosas letras.

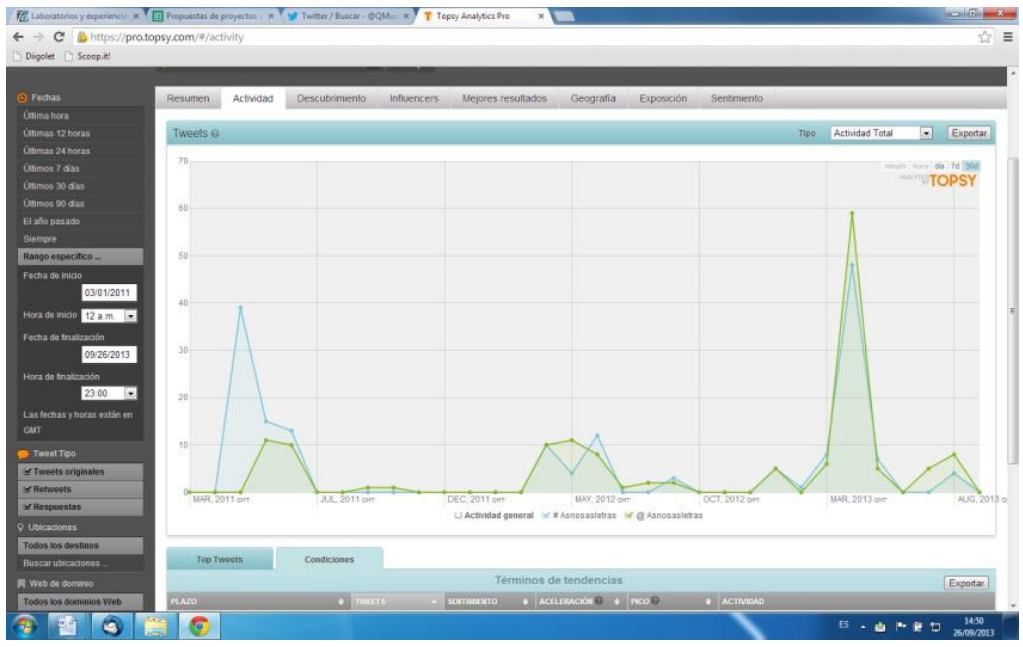

Fuente: Elaboración propia.

El proyecto colaborativo El Barco del Exilio dio comienzo en noviembre de 2012, y gracias a la investigación tenemos conocimiento de que todavía está en funcionamiento. Su índice de exposición está muy por encima de la media de los proyectos colaborativos estudiados, que corresponde a 1250, conseguido entre agosto y septiembre de 2013. La actividad de su hastag \#exilioyregreso no coincide con la de la cuenta del proyecto en Twitter. En la siguiente tabla se puede apreciar una comparación entre la actividad del hastag y la del proyecto:

Cuadro ${ }^{0}$ 9. Tabla comparativa entre el hastag y la cuenta de Twitter del proyecto El Barco del Exilio.

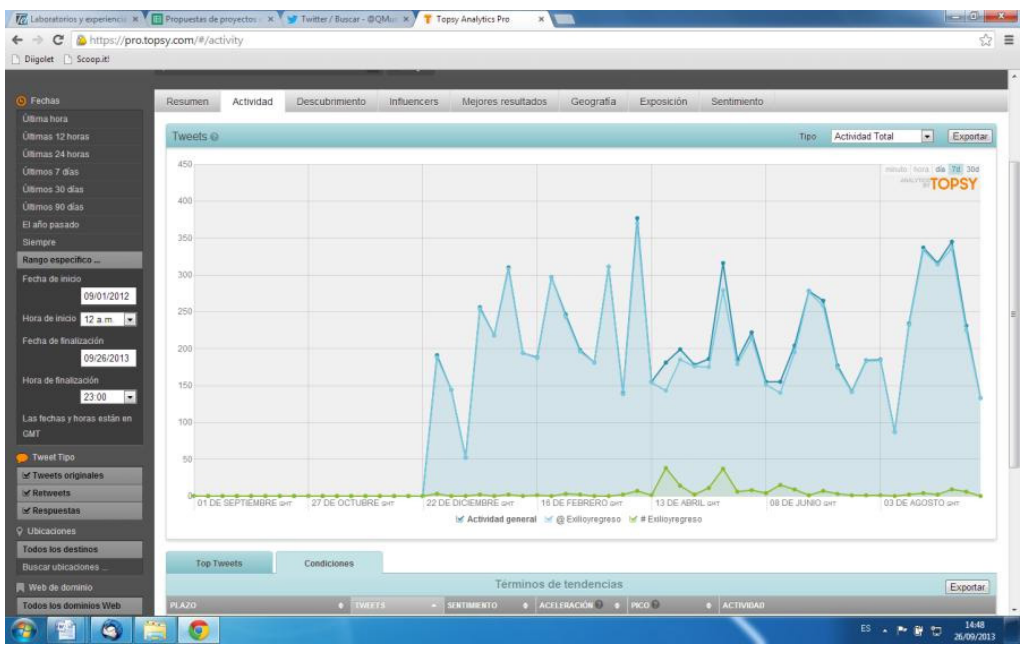

Fuente: elaboración propia. 
El proyecto colaborativo Ortografía y cómic dio comienzo en septiembre de 2012, y gracias a la investigación tenemos conocimiento de que todavía está en funcionamiento. Su índice de exposición está muy por debajo de la media de los proyectos colaborativos estudiados, que corresponde a 26 , conseguido entre octubre y noviembre de 2012 y la segunda vez entre marzo y abril de 2013. Este proyecto no tiene hastag.

Cuadro $\mathbf{n}^{\mathbf{0}}$ 10. Tabla de la actividad de la cuenta de Twitter del proyecto ortografía y Cómic.

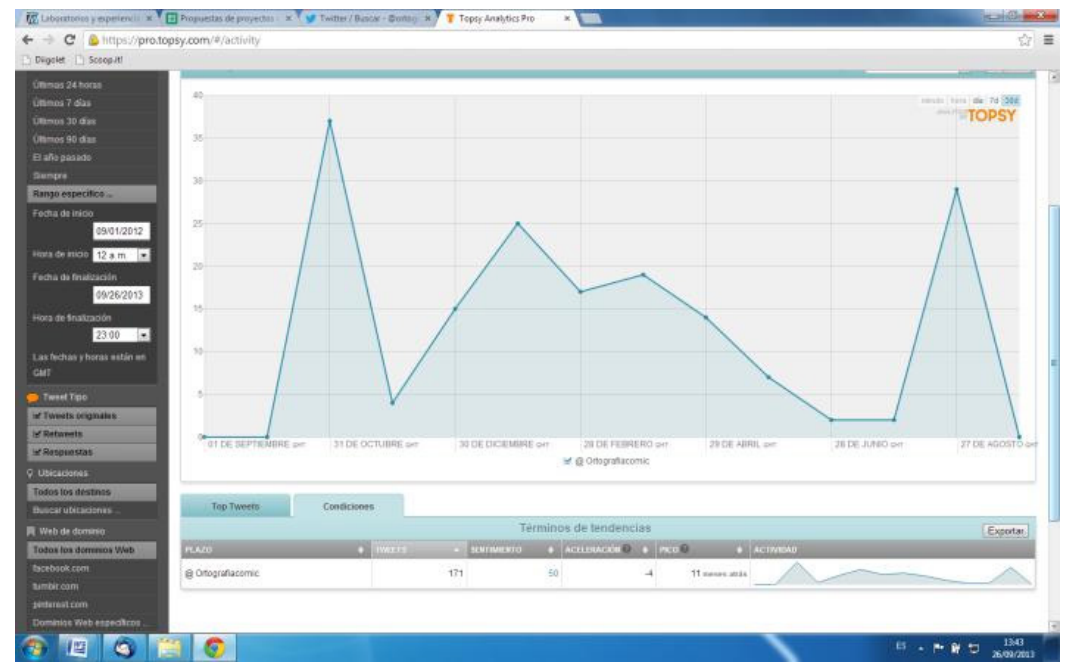

Fuente. Elaboración propia.

El proyecto colaborativo FP en tu Móvil dio comienzo en septiembre de 2011, y gracias a la investigación tenemos conocimiento de que se encuentra inactivo desde junio de 2013. Su índice de exposición está muy por debajo de la media de los proyectos colaborativos estudiados, que corresponde a 37, conseguido entre diciembre de 2011 y enero de 2012. La actividad de su hastag \#fpentumóvil dio comienzo en noviembre de 2011, unos meses después de la cuenta de Twitter. En la siguiente tabla se puede apreciar una comparación entre la actividad del hastag y la del proyecto: 
Cuadro $\mathbf{n}^{0}$ 11. Tabla comparativa entre el hastag y la cuenta de Twitter del proyecto FP en tu Móvil.

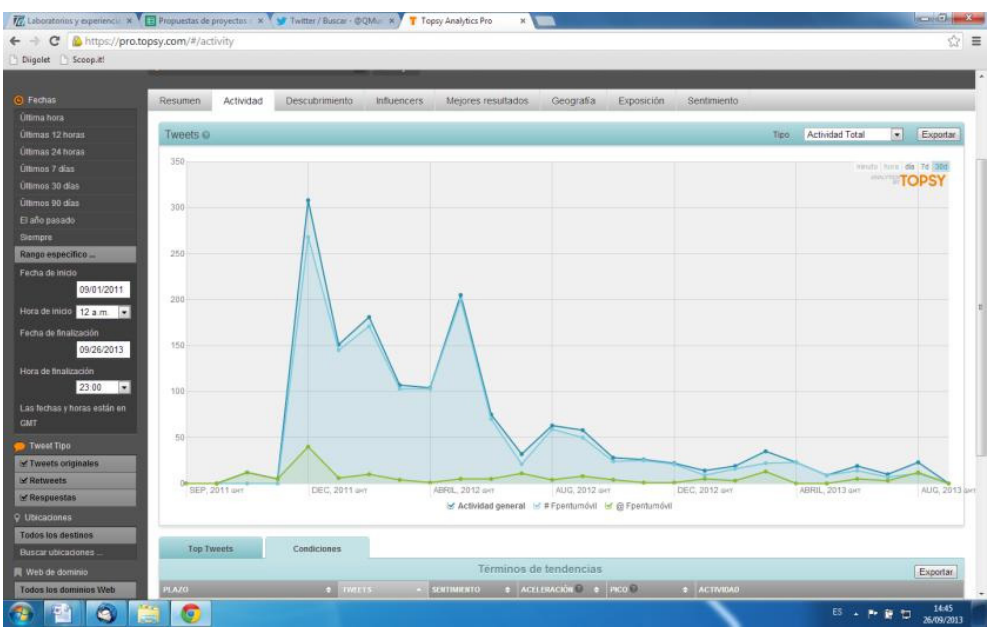

Fuente: elaboración propia.

El proyecto colaborativo Queremos MusicArte dio comienzo en noviembre de 2012, y gracias a la investigación tenemos conocimiento de que se encuentra activo actualmente. Su índice de exposición está por debajo de la media de los proyectos colaborativos estudiados, que corresponde a 126 , conseguido entre enero y marzo de 2013. La actividad de su hastag \#QMusicArte dio comienzo en noviembre de 2012, a la par que el proyecto. En la siguiente tabla se puede apreciar una comparación entre la actividad del hastag y la del proyecto:

Cuadro $\mathbf{n}^{\mathbf{0}}$ 12. Tabla comparativa entre el hastag y la cuenta de Twitter del proyecto Queremos MusicArte.

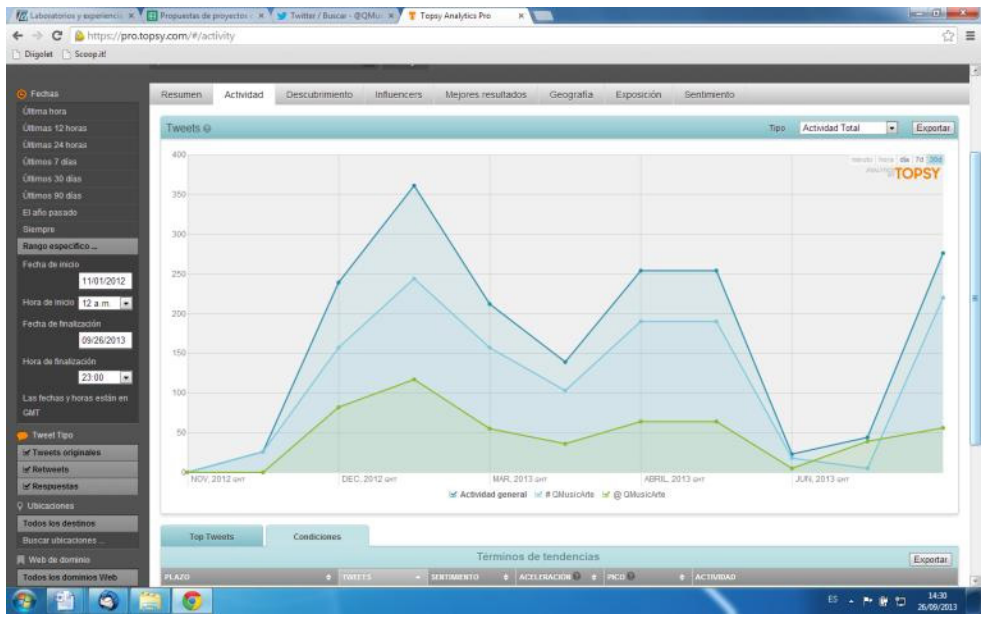

Fuente: elaboración propia. 
Es interesante comprobar los usuarios más activos en los proyectos colaborativos en red. Es así debido a que suele ser constante que los usuarios más influyentes en la red que siguen estos proyectos no son los que más actividad tienen dentro de los mismos, y en consecuencia, los que trabajan realmente para impulsar el proyecto. A continuación, podemos ver en la siguiente tabla los usuarios clave de los proyectos colaborativos examinados:

Cuadro $\mathbf{n}^{0}$ 13. Usuarios influyentes en los proyectos colaborativos investigados.

\begin{tabular}{|c|c|c|c|}
\hline $\begin{array}{l}\text { Nombre del } \\
\text { proyecto } \\
\text { colaborativo }\end{array}$ & $\begin{array}{l}\text { Seguidores más } \\
\text { influyentes }\end{array}$ & $\begin{array}{l}\text { Retuits más } \\
\text { influyentes de } \\
\text { seguidores }\end{array}$ & $\begin{array}{l}\text { Tuits más influyentes } \\
\text { de seguidores }\end{array}$ \\
\hline Espurn@ & $\begin{array}{l}\text { @.kurioso } \\
\text { @calameo } \\
\text { @jordi_a }\end{array}$ & $\begin{array}{l}\text { @educathyssen } \\
\text { @eraser } \\
\text { (a)balhisay }\end{array}$ & $\begin{array}{l}\text { @.carmebarba } \\
\text { @ mvertros } \\
\text { @ r.rbarlam }\end{array}$ \\
\hline Cero en Conducta & $\begin{array}{l}\text { @ queleer } \\
\text { @ Educacion3_0 } \\
\text { @) sandopen }\end{array}$ & $\begin{array}{l}\text { @1ondones } \\
\text { @ mjgsm } \\
\text { @,inmitacs }\end{array}$ & $\begin{array}{l}\text { @1ondones } \\
\text { @jhergony } \\
\text { @. mjgsm }\end{array}$ \\
\hline $\begin{array}{l}\text { Callejeros } \\
\text { Literarios }\end{array}$ & $\begin{array}{l}@ \text { eraser@carmen } \\
\text { martin } \\
@ \text { ainhoaeus }\end{array}$ & $\begin{array}{l}\text { @. lourdesdomenech } \\
\text { @,tonisolano } \\
\text { @,gorkafm }\end{array}$ & $\begin{array}{l}\text { @, lourdesdomenech } \\
\text { @,tonisolano } \\
\text { @,gorkafm }\end{array}$ \\
\hline Lóva & $\begin{array}{l}\text { @educaintef } \\
\text { @)radioclasica } \\
\text { @ leeres }\end{array}$ & $\begin{array}{l}@ \text { @starpy } \\
@ \text { miguelgilcasado } \\
@ \text {,mariaacaso }\end{array}$ & $\begin{array}{l}\text { @ proyectolova } \\
\text { @maryruthmcginn } \\
\text { @ doktus }\end{array}$ \\
\hline Kuentalibros & $\begin{array}{l}\text { @)misrecetas } \\
\text { @educaciontic } \\
\text { (a)leeres }\end{array}$ & $\begin{array}{l}\text { @starpy } \\
\text { @1ajaines @ } \\
\text { educandoando }\end{array}$ & $\begin{array}{l}\text { @ starpy } \\
\text { @educandoando } \\
\text { @)marudo76 }\end{array}$ \\
\hline As nosas letras & $\begin{array}{l}\text { @ranaencantada } \\
@ \text { mestraprimaria } \\
@ \text { silviagongo }\end{array}$ & $\begin{array}{l}@ \text { @estraprimaria } \\
@ \text { isabelmbs } \\
@ \text { o_fragha }\end{array}$ & $\begin{array}{l}\text { @) mestraprimaria } \\
\text { @isabelmbs } \\
@ \text { @o_fragha }\end{array}$ \\
\hline $\begin{array}{l}\text { El Barco del } \\
\text { Exilio }\end{array}$ & $\begin{array}{l}\text { @educaintef } \\
\text { @ roblesmaloof } \\
\text { @ideas4all }\end{array}$ & $\begin{array}{l}\text { @aidabsanchez } \\
\text { @eraser } \\
\text { @-minosita }\end{array}$ & $\begin{array}{l}\text { @exilioyregreso } \\
\text { @aidabsanchez } \\
\text { @eraser }\end{array}$ \\
\hline $\begin{array}{l}\text { Ortografía y } \\
\text { Cómic }\end{array}$ & $\begin{array}{l}\text { (@) eraser } \\
\text { @agirregabiria } \\
\text { (a) aulablog21 }\end{array}$ & $\begin{array}{l}\text { @ tonisolano } \\
\text { @enpiedelengua } \\
\text { @ pilarroman }\end{array}$ & $\begin{array}{l}\text { @.pilarroman } \\
\text { @aenpiedeaula } \\
\text { @) ortografiacomic }\end{array}$ \\
\hline FP en tu Móvil & $\begin{array}{l}\text { @ kevin_corbett } \\
\text { @educaredesp } \\
\text { @ tiscar }\end{array}$ & $\begin{array}{l}\text { @inedu } \\
@ \text { antonio_guirao } \\
@ \text { @mamenjg12 }\end{array}$ & $\begin{array}{l}\text { @)inedu } \\
\text { @.malonsorosa } \\
\text { @,iniciafp }\end{array}$ \\
\hline $\begin{array}{l}\text { Queremos } \\
\text { MusicArte }\end{array}$ & $\begin{array}{l}\text { @educathyssen } \\
\text { @eraser } \\
\text { (a) balhisay }\end{array}$ & $\begin{array}{l}\text { @ caminologa } \\
\text { @ mjgsm } \\
\text { @ o_fragha }\end{array}$ & $\begin{array}{l}\text { @ caminologa } \\
\text { @.inmitacs } \\
\text { @, mjgsm }\end{array}$ \\
\hline
\end{tabular}

Fuente: elaboración propia.

Como se puede apreciar, los índices de exposición determinan la influencia que el proyecto colaborativo ha tenidos sobre la comunidad educativa. Así pues, en la siguiente tabla podremos apreciar el ranking de proyectos colaborativos en red, y conocer cuál es el proyecto colaborativo con mayor influencia de los estudiados: 
Cuadro $\mathbf{n}^{\mathbf{0}}$ 14. Ranking de los proyectos colaborativos en red estudiados.

\begin{tabular}{|c|l|c|}
\hline Ranking & Nombre del proyecto colaborativo & Índice de exposición \\
\hline $1^{\circ}$ & El Barco del Exilio & 1250 \\
\hline $2^{\circ}$ & Lóva & 531 \\
\hline $3^{\circ}$ & Kuentalibros & 503 \\
\hline $4^{\circ}$ & Cero en Conducta & 366 \\
\hline $5^{\circ}$ & Espurn@ & 161 \\
\hline $6^{\circ}$ & Callejeros Literarios & 159 \\
\hline $7^{\circ}$ & Queremos MusicArte & 126 \\
\hline $8^{\circ}$ & As nosas letras & 59 \\
\hline $9^{\circ}$ & FP en tu móvil & 37 \\
\hline $10^{\circ}$ & Ortografía y Cómic & 26 \\
\hline
\end{tabular}

Fuente: elaboración propia.

La mayor parte de los proyectos colaborativos estudiados tienen un alcance nacional, pero el proyecto con más influencia El Barco del Exilio, cuenta con un avance mayor, involucrando a países de Latinoamérica.

Cuadro $\mathbf{n}^{0}$ 15. Influencia de los proyectos a nivel mundial.

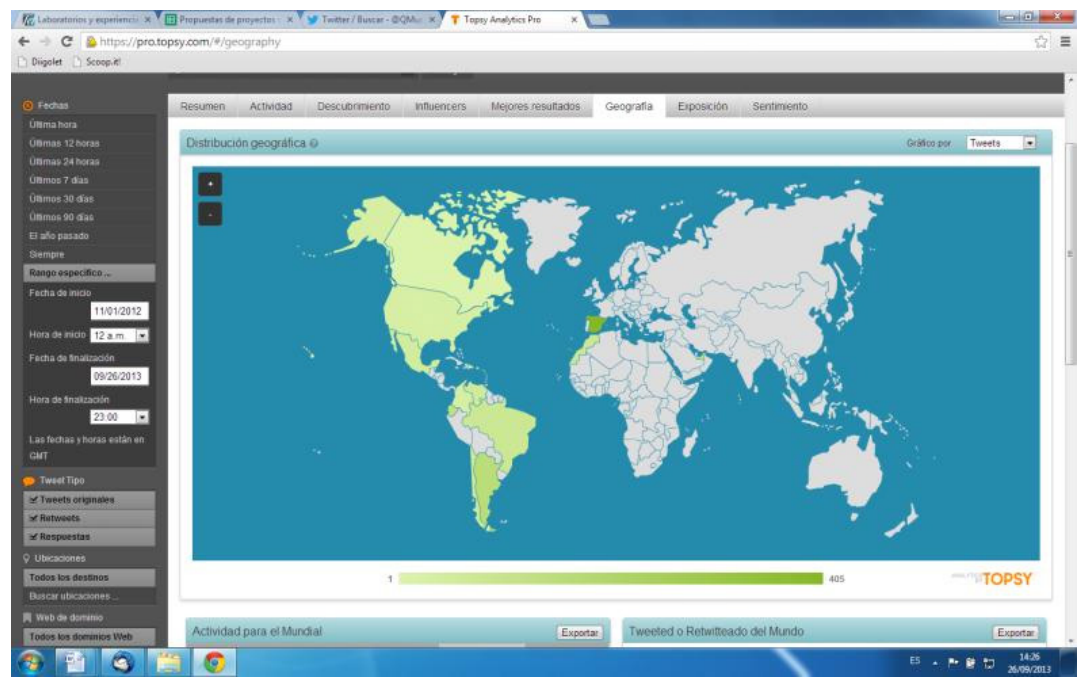

Fuente: elaboración propia. 
En esta investigación hemos estudiado 10 proyectos colaborativos en red que se encuentran activos en la fecha de la investigación. Gracias a los datos obtenidos, podemos obtener de ellos suficiente información como para esbozar unas conclusiones que determinen las causas de la influencia de los mismos en la comunidad docente que sigue su actividad.

Como se puede apreciar, el Barco del Exilio es el proyecto colaborativo en red con mayor influencia sobre la comunidad educativa, y que a su vez implica una actividad muy alta de sus usuarios.

\section{EI Barco del Exilio}

El proyecto El Barco del Exilio es una iniciativa que comenzó el profesor Joaquín José Martínez Sánchez, docente en la Comunidad Autónoma de Andalucía. Creó el proyecto colaborativo movido por la necesidad de tratar la situación actual de España en el aula, caracterizada por una amplia cantidad de jóvenes formados obligados a exiliarse del país para poder labrarse un futuro. Teniendo en cuenta esta realidad, creó un espacio de trabajo online desde el cual motivar a los demás docentes a trabajar este tema en el aula, compartiendo los resultados de esas buenas prácticas a través de las redes sociales.

El proyecto en sí, cuenta con actividad en más de 10 redes sociales, como Facebook, Linkedin o Proyéctate, entre otras. Su finalidad es poder llegar al mayor número de docentes, sin importar la red social en la que se encuentren activos.

A partir dela investigación realizada, como investigadoras hemos querido conocer el punto de vista del creador de este proyecto para que nos indique cuáles, a su criterio, son las razones de esta gran influencia que ha conseguido en la comunidad educativa. A continuación, podemos leer sus palabras:

"Creo que estamos recogiendo el fruto de un esfuerzo coordinado por traspasar las fronteras que separan escuela y social media, redes de docentes y aprendices de por vida, círculos enfocados sobre la tecnología educativa y las nuevas tendencias (educación expandida, aprendizajes invisibles, mobile learning, flipped learning, MOOC), con redes transversales que promueven una reforma pedagógica en profundidad. Hemos sacado provecho de la temática, en la medida que el exilio ha vuelto a estar de actualidad en nuestro país, a causa de la sangría de jóvenes que se ven forzados a salir en busca de trabajo, muchas veces por debajo de su preparación. Otros jóvenes, todavía escolarizados, pueden percibir la cercanía vivencial entre distintas épocas, caracterizadas por exilios masivos, desde la expulsión de judíos y moriscos hasta la emigración de los años 60/70 y el actual exilio juvenil. Hemos creado un transmedia sobre los exilios, para que niños y jóvenes participen de esa memoria cultural y biográfica, a la vez que la recrean y la reinventan. 
Además, el tema multipolar del exilio nos ha ido aproximando y enredando, sucesivamente, con personas y redes que hacen memoria de sus valores en América Latina (años 70-90), con migrantes y organizaciones sociales que comparten su empatía hacia las grandes migraciones de Sur a Norte: de Mesoamérica a USA, de África a España. La situación política y económica de sus países de origen justificaría en muchas ocasiones que se les reconociera la condición de refugiados, aunque no la soliciten. Un dato relevante: España es el país de Europa que, proporcionalmente, admite menos refugiados. Se prefiere obviar el carácter forzoso de la mayor parte de las migraciones, por pura economía de medios. Es una forma de ignorancia, un déficit educativo que nos hace (o nos hacía) sordos. Quien no se plantea problemas no puede encontrar soluciones, ni aun en su propia casa.

El proyecto surge con el objetivo genuino de promover la innovación educativa en métodos y herramientas, en la medida que permiten transformar el currículum, acreditar a los aprendices, hacer frente al abandono escolar y mejorar la calidad de los aprendizajes: creatividad, conectividad, sentido crítico, resolución de problemas concretos. La vinculación desde el origen entre la opción por un cambio de valores, un currículo abierto para una nueva sociedad conectada y las prácticas de excelencia con equidad, que se han construido en el Barco durante este primer año, nos han dado confiabilidad entre docentes activos y personas con un compromiso social. Quizá haya provocado alguna alarma a quienes no desean establecer ese tipo de alianzas, ni derribar los muros que nos separan. Pero hemos demostrado que en el Barco cabemos todos: somos hijos y madres/padres de culturas creadas en el exilio. Nuestra preocupación por el presente y el futuro es veraz. Las soluciones que ofrecemos (incluyo al magnífico equipo que se ha reunido en apenas diez meses) son viables. Lo serían todavía más sin los tabúes que frenan la innovación y los recortes que la privan de financiación.

Frente a la crisis, y a contracorriente, nos hemos inventado el \#nMOOC: un curso abierto no masivo, que aprovecha las pautas del conectivismo para generar comunidades de práctica y experiencia. Ofrecemos lo poco que sabemos, esperando que se multiplique en las relaciones que establecen las redes: Twitter, FB, Linkedin, la red especializada Proyéctate, Wordpress y hasta 30 escenarios digitales. Queremos ser "cualquiera" que tenga algo que dar y que recibir gratuitamente, mientras se reducen los presupuestos, se desmantelan espacios tan relevantes como Internet en el Aula, se dedican esfuerzos y capital desporporcionados a prácticas que no innovan, ni comunican, ni conectan.

Alguna de esas causas o todas ellas juntas explican los datos que habéis analizado."

\section{Conclusiones}

Esta investigación concluye con el reconocimiento como proyecto colaborativo más influyente de los estudiados a El Barco del Exilio. Recordemos que de los 82 
proyectos colaborativos registrados en el documento de Curalia que inició el profesor Gregorio Toribio, 10 son los que actualmente se mantienen activos. Entre estos 10 existe una media de 321 como índice de exposición en red, siendo solo cuatro los proyectos colaborativos que la superan.

Para poder visualizar el proyecto de forma gráfica y cercana, hemos creado una imagen que contiene realidad aumentada. Si es escaneada con el programa Layar, podrán obtenerse los enlaces directos a los tras proyectos colaborativos determinados como más influyentes en la comunidad educativa en Twitter.

Cuadro $\mathbf{n}^{0}$ 16: Resumen y conclusiones del trabajo de investigación.

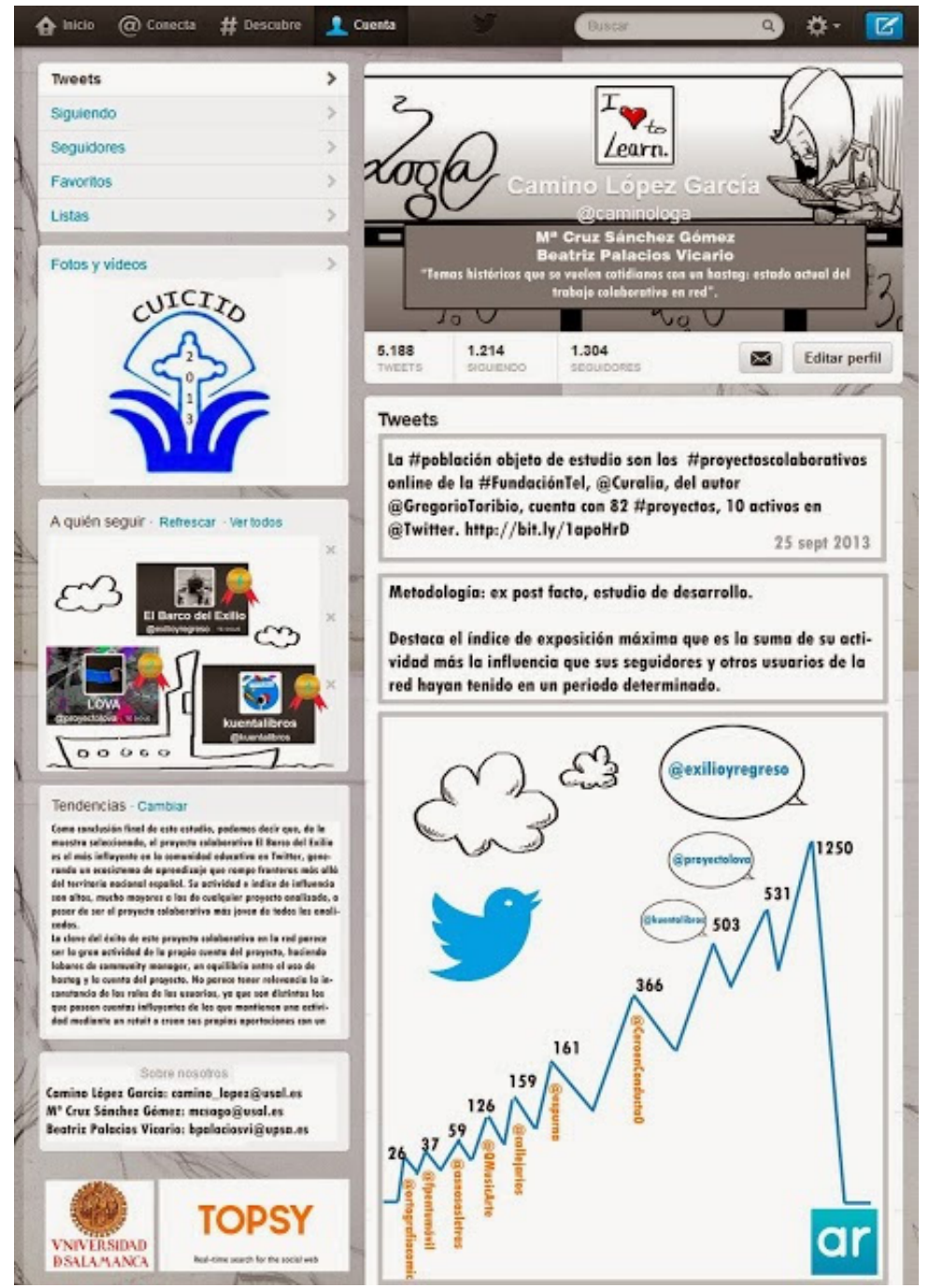

Fuente: elaboración propia. 


\section{Bibliografía}

CABERO, J. (coord.) (1994). Investigaciones sobre la informática en el centro. Barcelona. PPU.

BRUNS, A.; BURGESS, J. (2011) "The use of Twitter in the formation of Ad Hoc Publics". Comunicación presentada en la European Consortium for Political Research conference, Reiliavik, 25-27 de agosto. [Consultado el: 25/09/2013] [Disponible en http://snurb.info/files/2011/The\%20Use\%20of\%20Twitter\%20 Hashtags \%20in\%20the \%20Formation \%20of\%20Ad\%20Hoc\%20Publics $\% 20$ (final).pdf]

CASTAÑEDA, L.; ADELL, J. (2011) “El desarrollo profesional de los docentes en entornos personales de aprendizaje (PLE)". La práctica educativa en la sociedad de la información: innovación a través de la investigación. En Roig Vila, R. y Laneve, C. (Eds) Alcoy: Marfil, 83-95. [Consultado el 25/09/2013] [Disponible en http://digitum.um.es/xmlui/bitstream/10201/24647/1/CastanedaAdell2011preprint.pdf]

CHURCES, R. (2009). PNL para profesores: Cómo ser un profesor altamente eficaz. Bilbao: Desclee Bruwer.

COBO, C.; MORAVEC. J. (2011) Aprendizaje invisible. [Consultado el 25/09/2013] [Disponible en http://www.invisiblelearning.com/libro.html]

CURALIA (2013) Proyectos colaborativos. Laboratorios y experiencias. Fundación Telefónica. [Consultado el 25/09/2013] [Disponible en: http://laboratorios.fundaciontelefonica.com/blog/2013/01/10/proyectos-colaborativos/]

FOWLER, J. (2011) El poder de las redes sociales. RTVE. Transcripción [Consultado el 25/09/2013] [Disponible en: http://www.rtve.es/television/20110403/poder-redes-sociales/421888.shtml]

FREIRE, J. (2011). Ecosistemas de Aprendizaje. Ponencia en el Congreso Ideas worth spreading de la Universidad Internacional Méndez Pelayo. [Consultado el 25/09/2013] [Disponible en http:/www.youtube.com/watch?v=w7QOERmeG9s]

MEIRINHOS, M. Y ASÓRIO, A. (2009). "Las comunidades virtuales de aprendizaje: el papel central de la colaboración”. En: Pixelbit. Revista de medios y educación, $n^{\circ} 35,45-60$.

SIEMENS, G. (2004) Connectivism: A Learning Theory for the Digital Age. Elearning Espace. [Consultado el 25/09/2013] [Disponible en: http://www.ingedewaard.net/ papers/connectivism/2005_siemens_ALearningTheoryForTheDigitalAge.pdf]

ZAHO, J.; OTROS (2002). Factors affecting technology uses in schools: an ecological perspective. [Consultado el 25/09/2013] [Disponible en: https://www.msu. edu/ kenfrank/papers/Factors\%20affecting\%20technology $\% 20$ uses $\% 20 \mathrm{in} \% 20$ schools.pdf]

\section{Notas}

1. Para profundizar en el conocimiento del proyecto más influyente de la red, El Barco del Exilio, pueden consultar su web en Google Sites como El Barco del Exilio, guía de viaje. 


\section{Las autoras}

Camino López García es Licenciada en Bellas Artes, Máster en Profesor y Máster TIC en Educación. Actualmente está realizando su tesis doctoral en la Universidad de Salamanca acerca del trabajo colaborativo en red y los entornos personales de aprendizaje. Trabaja como Orientadora Tecnológica en el Centro Internacional de Tecnologías Avanzadas, (CITA) y es profesora de cursos online de este organismo y del INTEF.

Ma Cruz Sánchez Gómez es Doctora y Licenciada en Ciencias de la Educación en la Universidad de Salamanca y Máster en Logopedia en la Universidad Pontificia de Salamanca. Profesora Titular del Departamento de Didáctica, Organización y Métodos de Investigación de la Universidad de Salamanca con perfil en métodos de investigación y diagnóstico en educación (2 sexenios). Sus áreas de trabajo están relacionadas con las Nuevas Tecnologías (investigadora de grupo de Excelencia), colectivos con riesgo de exclusión social (coordinadora de las investigaciones relacionadas con la mujer y discapacidad del INICO) y evaluación educativa (con miembros de MIDE). En 2008 recibió primer el Primer Premio Nacional de Investigación de la Obra Social Caja Madrid. Directora del Servicio de Formación Continua de la Universidad de Salamanca (2007/2010).

Beatriz Palacios Vicario es Doctora en Psicología, Licenciada en Psicopedagogía pos la Universidad Pontificia de Salamanca y diplomada en Magisterio en Lengua Extranjera por la Universidad de Cantabria. Es profesora Encargada de Cátedra en el Departamento de Metodología de la investigación aplicada a las CC. del Comportamiento de la Facultad de Psicología de la Universidad Pontificia de Salamanca. Imparte docencia en la Facultad de Psicología y en las CC de la Actividad Física y Deportiva en asignaturas relacionadas con la metodología, así como en el Máster de Investigación y Máster del Profesorado de Educación Secundaria, Formación Profesional de la UPSA, y en el Máster Universitario en Estudios Avanzados sobre el Lenguaje, la Comunicación y sus Patologías. Sus líneas de investigación están relacionadas con el ámbito de la innovación educativa y género. 